\title{
Superhydrophobic and Superoleophilic Boron Nitride Nanotube-Coated Stainless Steel Meshes for Oil and Water Separation
}

\author{
Yuanlie Yu, Hua Chen,* Yun Liu, Vincent Craig, Lu Hua Li, and Ying Chen
}

Water pollution from many manufacturing industries, such as petrochemical, food, textiles, leather and metallurgical industries, is a major global problem. ${ }^{[1]}$ Polluted water often contains insoluble oil and harmful soluble pollutants that could easily decompose to other toxic chemicals, further polluting the natural environment and threatening aqueous habitats and human health. ${ }^{[2]}$ The separation and purification of water from polluted water is a current worldwide challenge that requires new technical and scientific breakthroughs. One of main issues in this field is to remove the oil contaminant from the ever-increasing oil/water mixtures produced by industry. Although several approaches were developed for this purpose, such as oil skimmers, centrifuges, coalescence, settling tanks, depth filters, magnetic separations, flotation technologies, oilabsorbing materials and combustion, ${ }^{[3]}$ it has not been drawn extremely considerable attention until Feng et al. reported a superhydrophobic and superoleophilic nanostructured coating on a stainless steel mesh for oil/water separation. ${ }^{[4]}$ This type of nanostructured coating materials with an unique surface wettability (originating from their superhydrophobic/superoleophilic or superhydrophilic/superoleophobic nature) enables a simple and efficient separation of oil from water through either filtration or absorption of oil or water in mixtures. ${ }^{[3 a, b, 5]}$ Recently, Hayase et al. reported the formation of a marshmallow-like gel from various types of alkoxysilanes (i.e., tri-, di-functional, and bridged alkoxysilanes). ${ }^{[6]}$ The gel behaves like a sponge, absorbing and removing organic compounds from water and then releasing the compounds when squeezed. Gao et al. also

Dr. Y. L. Yu, Dr. H. Chen

Centre for Advanced Microscopy

Australian National University

Canberra, ACT 0200, Australia

E-mail: hua.chen@anu.edu.au

Tel/Fax: +61 (0)2 61256886

Prof. Y. Liu

Research School of Chemistry

Australian National University

Canberra, ACT 0200, Australia

Prof. V. Craig

Department of Applied Mathematics

Research School of Physics and Engineering

Australian National University

Canberra, ACT 0200, Australia

Dr. L. H. Li, Prof. Y. Chen

Institute for Frontier Materials

Deakin University

Waurn Ponds Campus, Geelong, VIC 3216, Australia fabricated a double-layered copper mesh with micro- and nanostructured $\mathrm{TiO}_{2}$ on one layer and octadecyl phosphonic acid modified $\mathrm{TiO}_{2}$ on another layer, which leads to superhydrophobicity and superoleophilicity. ${ }^{[7]}$ Such a mesh can not only separate insoluble oil from water but also degrade the soluble pollutants in water under the irradiation of ultraviolet light due to the photocatalytic properties of $\mathrm{TiO}_{2}$. However, these materials require complicated and expensive chemical decoration/ fabrication processes. Additionally there are big concerns about their stability, selectivity and recyclability which block their practical applications. ${ }^{[8]}$

It is noteworthy that BNNTs possess good mechanical performance, high thermal conductivity, outstanding electrical insulation, as well as excellent chemical stability and superb oxidation resistance. ${ }^{[9]}$ These unique properties make BNNTs promising for applications as biological probes, ${ }^{[10]}$ and in optoelectronic devices, ${ }^{[11]}$ humidity sensing systems and as a hydrogen storage media, ${ }^{[12]}$ especially in hazardous and high-temperature environments. It is also found that as-fabricated BNNT films are unwetted by water due to their nanometer scale roughness as well as adsorbed hydrocarbon contaminations. ${ }^{[13]}$ This property can be enhanced if the BNNTs are partially and vertically aligned in which case they provide much better water repellency with a water contact angle (CA) over $150^{\circ}$ and less CA hysteresis, and higher stability at different $\mathrm{pH}$ values. ${ }^{[14]}$ More importantly, the as-fabricated BNNT films are superhydrophobic and superoleophilic without any further modification. This will advance their applications relative to other inorganic nanomaterials that need to be modified by the low surface energy organic chemicals since the organic chemicals may be dissolved by the oil, losing the superhydrophobicity. ${ }^{[7]}$ Furthermore, in terms of BNNTs' excellent chemical stability and high temperature oxidation-resistance (up to $900{ }^{\circ} \mathrm{C}$ in ambient environment), a BNNT layer could be an attractive candidate as a superhydrophobic surface in harsh chemical and thermal conditions.

In the study reported herein, we developed a simple, inexpensive and scalable approach that can be used to directly grow highly dense and pure BNNTs on the surface of a stainless steel mesh via a vapor-liquid-solid (VLS) growth process. The process was realized via a special process that is similar to ball milling and subsequent high temperature annealing, as reported in our previous works (see the Experimental section). $\left.{ }^{9 g}, 11,12 \mathrm{a}, 15\right]$ In addition, the hydrophobicity and oleophilicity of the BNNTcoated mesh as well as its selectivity and efficiency of oil/water separation were investigated.

Figure 1a,b presents typical field-emission scanning electron microscopy (FESEM) images of the uncoated and BNNTcoated mesh. It is apparent that BNNTs are homogeneously

DOI: 10.1002/admi.201300002 


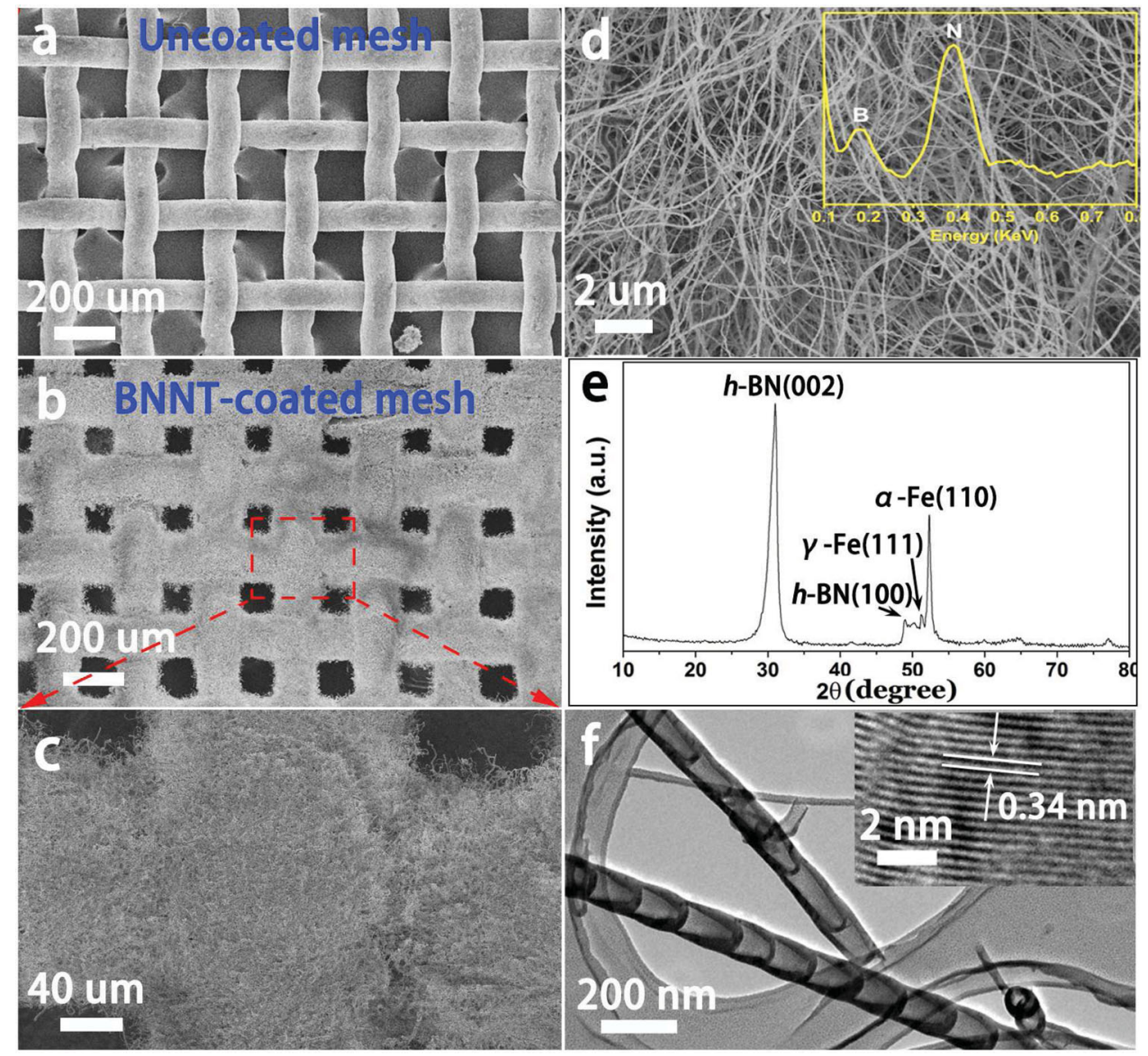

Figure 1. FESEM images of the a) uncoated and b) BNNT-coated stainless steel meshes, c) the BNNTs on the mesh surface, d) the corresponding peel-off BNNTs. e) XRD pattern and f) TEM image of the BNNTs. The insets in (d) and ( $f$ ) are the EDS spectrum and high-resolution TEM image of the BNNTs, respectively.

distributed on the mesh surface, decreasing the hole size of the mesh from the original $150 \mu \mathrm{m}$ down to $\approx 50-100 \mu \mathrm{m}$, and correspondingly, increasing the grid thickness from $\approx 70 \mu \mathrm{m}$ to over $100 \mu \mathrm{m}$. Most importantly, the BNNT coating reduces the hole size but does not completely block the holes, ensuring that wettable liquid passes quickly through the mesh. Figure 1c and $\mathrm{d}$ indicate that a large quantity of BNNTs with a diameter ranging from 100 to $400 \mathrm{~nm}$ are uniformly and randomly distributed on the surface of the grids to form a structure that ensures a large surface contact area required for highly efficient oil/water separation as discussed later. The metal elements in the stainless steel mesh act as a catalyst for the growth of BNNTs, ${ }^{[13 a]}$ therefore the nanotubes are firmly attached to the mesh surface, providing a strong adhesion of the BNNT to the mesh. Figure 1d indicates long BNNTs grow largely perpendicularly to the surface or the side face of the mesh, which not only benefits the water repellency but also enables controlling the pore size of BNNT-coated mesh. The inset in Figure 1d is the X-ray energy dispersive spectrum (EDS) of BNNTs, showing mainly boron and nitrogen. Figure 1e is a typical X-ray diffraction (XRD) pattern of the BNNT-coated mesh exhibiting two main diffraction peaks with d-spacing of 0.336 and $0.215 \mathrm{~nm}$ are identified to be interlayer distances of the (002) and (100) planes of the hexagonal boron nitride $(h$-BN) structure (JCPDF card No. 73-2095). ${ }^{[16]}$ Figure if shows a typical transmission electron microscopy (TEM) image of the BNNTs that were physically removed from the BNNT-coated mesh, revealing their bamboo-like character. The plane spacing of the BNNT walls is calculated to be $0.34 \mathrm{~nm}$ from the inset high-resolution TEM image of Figure 1f, corresponding to the (002) plane spacing of $h$-BN.

Figure 2 exhibits the wettability of the BNNT-coated and uncoated meshes to water and oil (exemplified via hexadecane). Figure 2a shows several water droplets resting on the BNNTcoated mesh with very high CAs, while the oil droplets rapidly spread and penetrate the BNNT-coated mesh (Figure 2b) as soon as they touch the upper surface of the coated mesh, clearly indicating the significant hydrophobic and oleophilic features of the BNNT-coated meshes. Figure $2 \mathrm{c}$ shows the shapes of the water droplets on an uncoated steel mesh at a tilted angle of $0^{\circ}$ and $180^{\circ}$. Noticeably, the CA of a water droplet on the surface of the uncoated mesh is approximately $115^{\circ}$. The water droplet still maintains a semispherical shape without obvious distortion when the uncoated mesh is gradually tilted to $180^{\circ}$, indicating that the uncoated mesh has a high CA hysteresis with respect to water droplets. Figure 2d,e shows the shapes of 


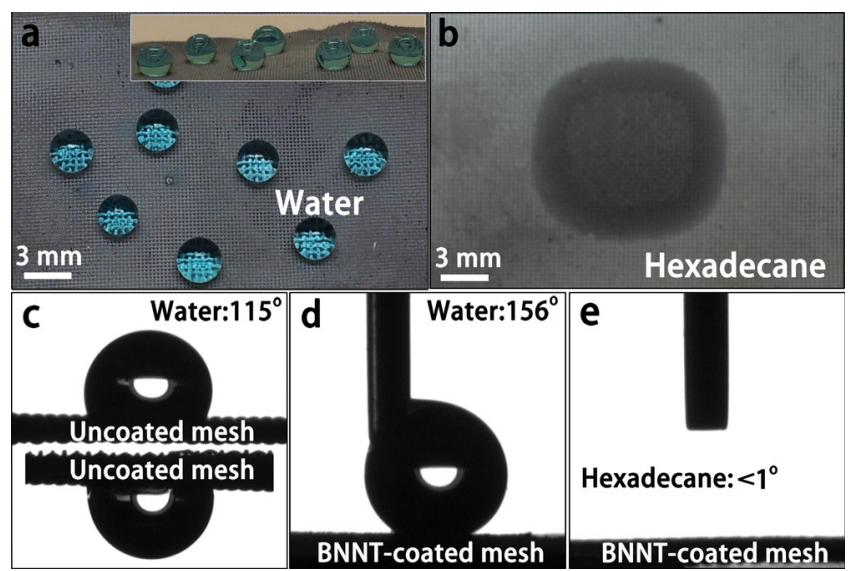

Figure 2. a) Photograph of water droplets (dyed with methylene blue) on the surface of the BNNT-coated mesh (inset: a side view of a water droplet); b) photograph of an oil droplet completely spread on the coated mesh surface; c,d) shapes of a water droplet $(6 \mu \mathrm{L})$ staying on the uncoated (up: no tilting, down: after tilting $180^{\circ}$ ) and coated mesh with a water CA of $115^{\circ}$ and $156^{\circ}$, respectively; e) photograph of the hexadecane droplet on the coated mesh surface with a CA of nearly zero.

individual water and hexadecane droplet on the surface of the BNNT-coated mesh, respectively. In this case, the water droplet shows an advancing water $\mathrm{CA}$ of $156^{\circ}$ and a receding water CA of $155^{\circ}$. The difference in advancing and receding CA is very small. We can therefore conclude that the BNNT-coated meshes have almost no hysteresis for a water droplet. As a result, the water droplets will spontaneously roll off if the mesh is slightly tilted (Figure S1 and Video 1, Supporting Information). The BNNT coating therefore plays an important role in the improvement of hydrophobicity of the mesh. On the other hand, the hexadecane droplet shows a CA of less than $1^{\circ}$ on the surface of BNNT-coated mesh (Figure 2e), demonstrating its strong oleophilic nature. Generally, the wettability of a solid surface mainly depends on its surface chemistry and the geometrical roughness. ${ }^{[17]}$ Lee et al. has discovered that the surface morphology of the BNNT layer has an important impact on the water CA. ${ }^{[14]}$ It is found that the surfaces with partially vertical aligned BNNTs exhibit excellent water repellency with a CA of over $150^{\circ} .{ }^{[14]} \mathrm{As}$ shown in Figure 1c (the enlarged view on the BNNT-coated mesh), the resultant BNNTs are grown on the surface of the mesh surface with partially vertical alignment and therefore exhibit a larger water CA. The combination of such superhydrophobic and superoleophilic effects makes the BNNT-coated mesh a highly suitable material for oil/water separation.

The BNNT-coated mesh, having excellent superhydrophobic and superoleophilic features, is thus expected to enable the separation of insoluble oil from water. In order to test this, an oil/ water separation experiment was setup as shown in Figure 3. The uncoated and BNNT-coated meshes, acting as the separation membrane, were placed between two glass tubes (with a well-polished flat surface to provide an excellent seal). Both the mesh and tubes were clamped together. The water, hexane and hexadecane as well as their mixtures (hexane/water and hexadecane/water) were used to demonstrate associated effects. The hexane and hexadecane can easily pass through the uncoated
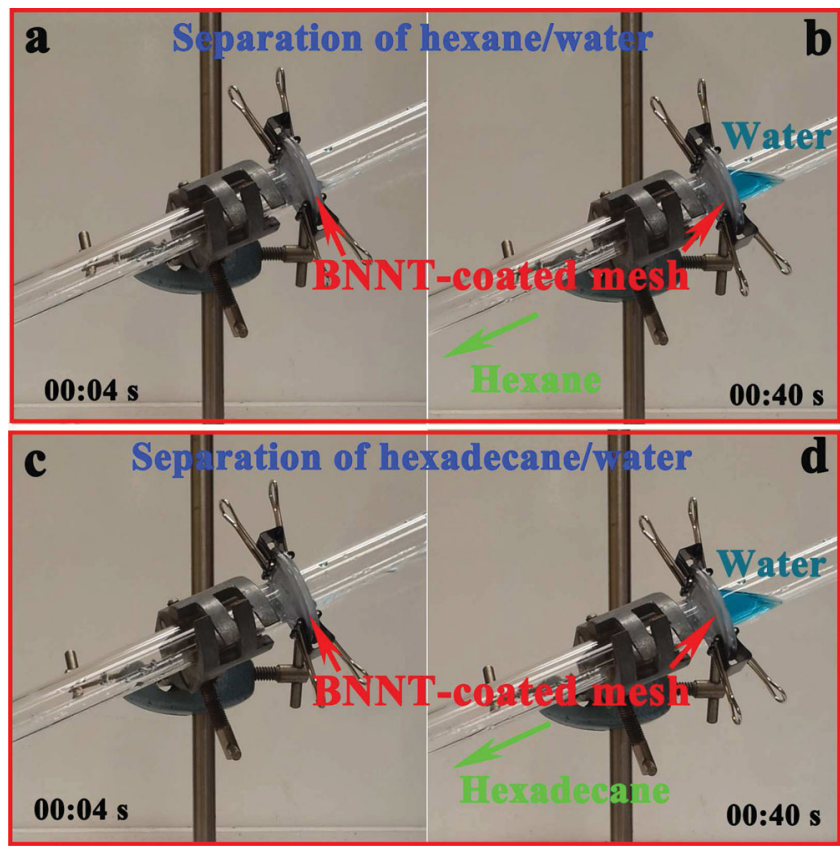

Figure 3. The experimental setup and the filtration/separation process of oil (hexane and hexadecane)/water. Water stays over the coated mesh, and hexane and hexadecane go through the coated mesh after filtration or separation (water is dyed using methylene blue to enhance the visual effect).

mesh under gravity and flow down quickly (Figure S2 and Video 2, Supporting Information). With the BNNT-coated mesh, however, the water remains above the mesh while the hexane and hexadecane easily pass through the mesh and flow down quickly (Figure S3 and Video 3, Supporting Information). Figure 3 shows the separation process of insoluble oil and water, where the tubes are set an oblique angle to ensure that the insoluble oil makes contact with the mesh. Then, the mixtures of $20 \mathrm{~mL}$ hexane/water and hexadecane/water (with a volume ratio of 18:2) are separately poured into the glass tube. It is seen that the hexane and hexadecane quickly penetrate through the coated mesh and drop into the beaker below due to the superoleophilicity of the BNNT-coated mesh while water (dyed by the methylene blue for the purpose of enhancing the visual effect) remains above the mesh because of the superhydrophobicity and low water-adhesion property of BNNT-coated mesh (Video 4 and 5, Supporting Information). It is found that the oil/water separation time increases with the increasing oil volume fractions for certain volume mixtures. In a designated experimental condition (where the quartz cylinder has a BNNT-coated mesh with an area of $\approx 1 \mathrm{~cm}^{2}$ ), the diffusion rate can be calculated to be $37.6 \mathrm{~L} / \mathrm{m}^{2} \mathrm{~s}$ for hexane and $16.2 \mathrm{~L} / \mathrm{m}^{2} \mathrm{~s}$ for hexadecane, respectively. The long chain hexadecane take more time to go through the mesh due to its higher viscosity. Figure 4 shows the images of the $20 \mathrm{~mL}$ oil/water mixture (with a volume ratio of 18:2) as well as of corresponding oil and water after the separation. Over $17.5 \mathrm{~mL}$ oil (hexane and hexadecane) and $1.9 \mathrm{~mL}$ water were collected, suggesting extremely high separation efficiency of the BNNT-coated mesh. More importantly, neither water nor oil is observed in collected oil or water, 


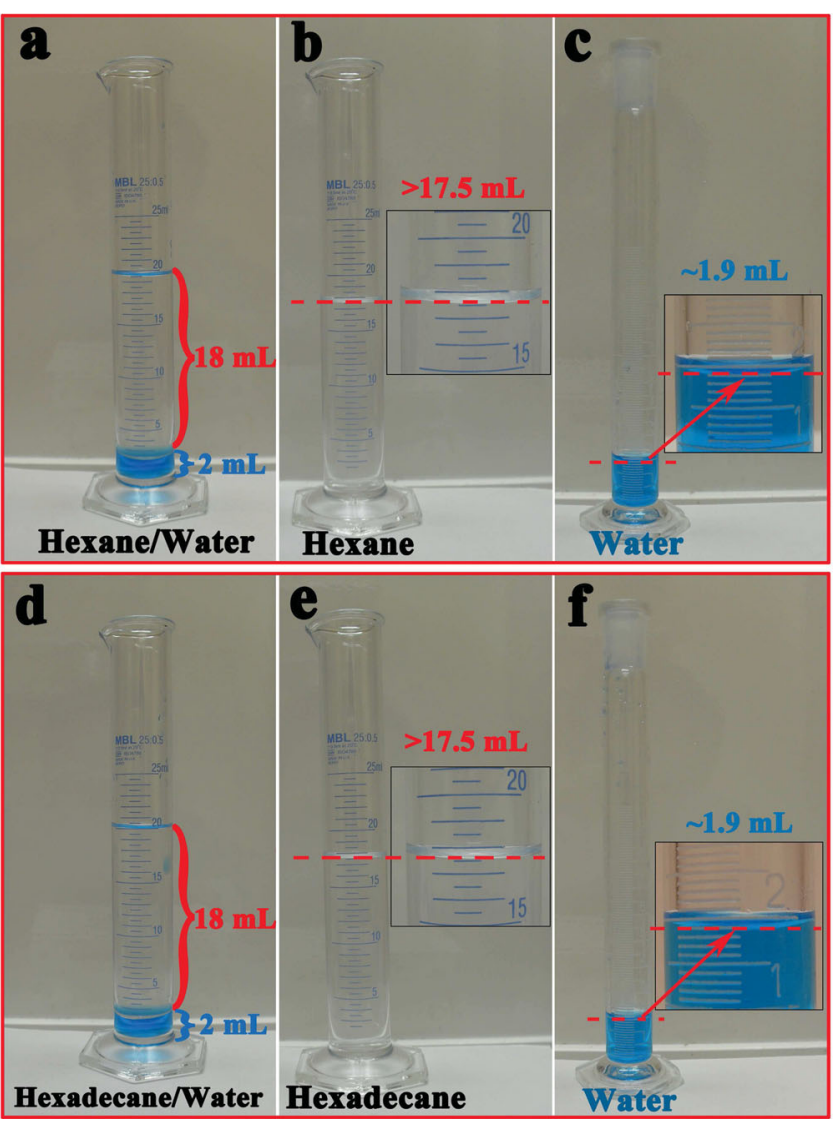

Figure 4. The photographs of the water/oil (hexane or hexadecane) mixtures $(a, d)$, oil $(b, e)$ and water $(c, f)$ collected after the separation.

respectively, indicating a high purity and effectiveness of the separation of the oil/water using the BNNT-coated mesh.

It is noteworthy that the BNNTs have one end attached to the surface as the iron in the stainless steel acts as a catalyst for the growth of BNNTs, ${ }^{[13 a]}$ and that they grow outward from this surface providing a certain degree of alignment. In such a way, BNNTs are strongly adhered to the stainless steel mesh. It is found that the efficiency of the oil/water separation of this BNNT-coated mesh remains the same after over 100 cycles without any noticeable deterioration. This suggests that the BNNT-coated mesh not only presents superhydrophobic and superoleophilic properties, but they also have the virtue of stability, durability and repeatability. These, in conjunction with the excellent mechanical and chemical stability of BNNTs, make the BNNT-coated stainless steel mesh a promising candidate for the treatment of oil-polluted water.

In summary, we have developed a simple, effective and scalable approach to fabricate a durable, reusable and reproducible BNNT-coated mesh that has superhydrophobic and superoleophilic properties. The BNNTs with diameters in the range of 100 to $400 \mathrm{~nm}$ are partially and vertically aligned on the surface of the stainless steel mesh, altering the surface properties of the stainless steel mesh from hydrophobicity with a high water CA hysteresis to superhydrophobicity and superoleophilicity without hysteresis. The BNNT-coated mesh developed in this work can therefore be practically used as a highly efficient filtration membrane for the separation of oil from water in oilpolluted water, benefiting the environment and human health.

\section{Experimental Section}

Preparation of BNNT-Coated Meshes: For a typical synthesis process, the amorphous boron powder was first ball-milled in a planetary mill with a sealed stainless steel container filled with $\mathrm{NH}_{3}$ gas for $50 \mathrm{~h}$ at a rotation speed of $300 \mathrm{rpm}$ at room temperature. The weight ratio of the steel balls to the boron powder was 50:1. After ball-milling, the milled boron powder was put into an iron boat and covered with a cleaned $2 \mathrm{~cm} \times 2 \mathrm{~cm}$ stainless steel mesh (that did not touch the powder). The mesh has the pore size of approximately $150 \mu \mathrm{m}$ (100 mesh). The resultant iron boat with this mesh cover was placed in a tube furnace and annealed at $1100{ }^{\circ} \mathrm{C}$ for $8 \mathrm{~h}$ under a $\mathrm{H}_{2} / \mathrm{N}_{2}\left(5 \% \mathrm{H}_{2}\right)$ gas flow $(100 \mathrm{ml} / \mathrm{min})$. In such a way, the BNNT-coated mesh was prepared via the in situ VLS growth model where the boron was evaporated and then BNNT grown up on the mesh surface with an assistance of iron element in the stainless steel mesh as a catalyst.

Preparation and Separation of the Oil/Water Mixtures: The water (dyed with methylene blue) and oil (hexane or hexadecane) with 1:9 volume ratio was firstly mixed and then strongly stirred for $10 \mathrm{~min}$. For every separation, a total $20 \mathrm{~mL}$ oil/water mixture was slowly poured into the glass tube, and separated by the BNNT-coated mesh.

Characterization: The morphology of the uncoated and BNNTcoated meshes was characterized by a field emission scanning electron microscopy (FESEM, Zeiss UltraPlus) using $1 \mathrm{kV}$ accelerating voltage and a 2-4 $\mathrm{mm}$ working distance. The chemical composition of BNNTs was estimated by energy dispersive spectrometer (EDS, INCA) attached to the FESEM with $15 \mathrm{kV}$ accelerating voltage and a $10.9 \mathrm{~mm}$ detecting distance. Transmission electron microscopy (TEM CM300, Philips), and

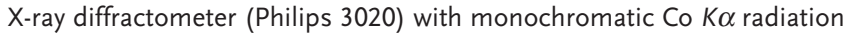
were employed to probe the structure of the as-synthesized BNNTcoated mesh. The surface wettability of the BNNT-coated mesh was characterized using KSV CAM200 Goniometer instrument at ambient temperature in air, and the water CA was determined by averaging measurements taken from at least five different positions on the measured surface.

\section{Supporting Information}

Supporting Information is available from the Wiley Online Library or from the author.

\section{Acknowledgements}

The authors acknowledge the financial supports from the Australian Microscopy and Microanalysis Research Facility (AMMRF) and from the Australian Research Council in the form of the Discovery Projects.

Received: August 13, 2013

Revised: September 23, 2013

Published online: November 8, 2013

[1] Handbook of Environmental Engineering (Eds: L. K. Wang, J. P. Chen, Y. T. Hung, N. K. Shammas), Humana Press, New York 2008, 13, 639.

[2] a) D. Dudgeon, A. H. Arthington, M. O. Gessner, Z. Kawabata, D. J. Knowler, C. Leveque, R. J. Naiman, A. H. Prieur-Richard, D. Soto, M. L. J. Stiassny, C. A. Sullivan, Biol. Rev. 2006, 81, 163; 
b) C. Wu, C. Maurer, Y. Wang, S. Xue, D. L. Davis, Environ. Health Perspect. 1999, 107, 251.

[3] a) W. B. Zhang, Z. Shi, F. Zhang, X. Liu, J. Jin, L. Jiang, Adv. Mater. 2013, 25, 2071; b) W. W Lei, D. Portehault, D. Liu, S. Qin, Y. Chen, Nat. Commun. DOI: 10.1038/ncomms2818; c) A. Bayat, S. F. Aghamiri, A. Moheb, G. R. Vakili-Nezhaad, Chem. Eng. Technol. 2005, 28, 1525; d) M. O. Adebajo, R. L. Frost, J. T. Kloprogge, O. Carmody, S. Kokot, J. Porous Mater. 2003, 10, 159.

[4] L. Feng, Z. Y. Zhang, Z. Y. Mai, Y. M. Ma, B. Q. Liu, L. Jiang, D. B. Zhu, Angew. Chem. Int. Ed. 2004, 43, 2012.

[5] a) Y. Z. Zhu, F. Zhang, D. Wang, X. F. Pei, W. B. Zhang, J. Jin, J. Mater. Chem. A 2013, 1, 5758; b) J. Zhang, S. Seeger, Adv. Funct. Mater. 2011, 21, 4699

[6] G. Hayase, K. Kanamori, M. Fukuchi, H. Kaji, K. Nakanishi, Angew. Chem. Int. Ed. 2013, 52, 1986.

[7] C. G. Gao, Z. X. Sun, K. Li, Y. N. Chen, Y. Z. Cao, S. Y. Zhang, L. Feng, Energy Environ. Sci. 2013, 6, 1147.

[8] Y. W. Shang, Y. Si, A. Raza, L. P. Yang, X. Mao, B. Ding, J. Y. Yu, Nanoscale 2012, 4, 7847

[9] a) A. P. Suryavanshi, M. Yu, J. Wen, C.C. Tang, Y. Bando, Appl. Phys. Lett. 2004, 84, 2527; b) D. Golberg, P. M. F. J. Costa, O. Lourie, M. Mitome, X. D. Bai, K. Kurashima, Nano Lett. 2007, 7, 2146; c) L. Wirtz, A. Rubio, Phys. Rev. B 2003, 68, 045425; d) D. Sanchez-Portal, E. Hernandez, Phys. Rev. B 2002, 66, 235415; e) V.N. Popov, Phys. Rev. B 2003, 67, 085408; f) A. Rubio, www.advmatinterfaces.de

J. F. Corkoll, M. L. Cohen, Phys. Rev. B 1994, 49, 5081; g) Y. Chen, J. Zou, S. J. Campbell, G. L. Caer, Appl. Phys. Lett. 2004, 84, 2430.

[10] a) G. Ciofani, V. Raffa, A. Menciassi, A. Cuschieri, Nano Today 2009, 4, 8; b) X. Chen, P. Wu, M. Rousseas, D. Okawa, Z. Gartner, A. Zettl, C. R. Bertozzi, J. Am. Chem. Soc. 2009, 131, 890.

[11] a) H. Chen, Y. Chen, C. P. Li, H. Z. Zhang, J. S. Williams, Y. Liu, Z. W. Liu, S. P. Ringer, Adv. Mater. 2007, 19, 1845; b) H. Chen, Y. Chen, Y. Liu, C. N. Xu, J. S. Williams, Opt. Mater. 2007, 29, 1295; c) L. H. Li, Y. Chen, M. Y. Lin, A. M. Glushenkov, B. M. Cheng, Appl. Phys. Lett. 2010, 97, 141104.

[12] a) Y. L. Yu, H. Chen, Y. Liu, L. H. Li, Y. Chen, Electrochem. Commun. 2013, 30, 29; b) R. Z. Ma, Y. Bando, H. W. Zhu, T. Sato, C. L. Xu, D. H. Wu, J. Am. Chem. Soc. 2002, 124, 7672.

[13] a) L. H. Li, Y. Chen, A. M. Glushenkov, J. Mater. Chem. 2010 20, 9679; b) L. H. Li, Y. Chen, Langmuir 2010, 26, 5135; c) L. Li, L. H. Li, S. Ramakrishnan, X. J. Dai, K. Nicholas, Y. Chen, Z. Q. Chen, X. W. Liu, J. Phys. Chem. C 2012, 116, 18334.

[14] C. H. Lee, J. Drelich, Y. K. Yap, Langmuir 2009, 25, 4853.

[15] a) H. Chen, Y. Chen, Y. Liu, L. Fu, C. Huang, D. Llewellyn, Chem. Phys. Lett. 2008, 463, 130; b) H. Chen, Y. Chen, Y. Liu, J. Alloys Compd. 2010, 504, 353.

[16] J. L. Wang, L. P. Zhang, G. W. Zhao, Y. L. Gu, Z. H. Zhang, F. Zhang, W. M. Wang, J. Solid State Chem. 2011, 184, 2478.

[17] L. Feng, S. Li, Y. Li, H. Li, L. Zhang, J. Zhai, Y. Song, B. Liu, L. Jiang, D. Zhu, Adv. Mater. 2002, 14, 1857. 